\title{
Pacific
}

Journal of

Mathematics

\section{THE VIRTUAL FIRST BETTI NUMBER OF SOLUBLE GROUPS}

MaRTIN R. BRIDSON AND Dessislava H. Kochloukova 


\title{
THE VIRTUAL FIRST BETTI NUMBER OF SOLUBLE GROUPS
}

\author{
MaRTIN R. BRIDSON AND Dessislava H. Kochloukova
}

\begin{abstract}
We show that if a group $G$ is finitely presented and nilpotent-by-abelianby-finite, then there is an upper bound on $\operatorname{dim}_{\mathbb{Q}} H_{1}(M, \mathbb{Q})$, where $M$ runs through all subgroups of finite index in $G$.
\end{abstract}

\section{Introduction}

The virtual first betti number of a finitely generated group $G$ is defined as

$$
\operatorname{vb}_{1}(G)=\sup \left\{\operatorname{dim} H_{1}(S, \mathbb{Q}) \mid S \leq G \text { of finite index }\right\} .
$$

A group is said to be large if it has a subgroup of finite index that maps onto a nonabelian free group. If $G$ is large then $\operatorname{vb}_{1}(G)=\infty$. It is easy to find finitely generated groups $G$ that are not large but have $\mathrm{vb}_{1}(G)=\infty$. For example, in the metabelian group $\mathbb{Z} \imath \mathbb{Z}=\langle a, t|\left[a, t^{-n} a t^{n}\right]=1$ for all $\left.n\right\rangle$, the subgroup $S_{m}<\mathbb{Z} \imath \mathbb{Z}$ generated by $t^{m}$ and the conjugates of $a$ has index $m$ and $H_{1}\left(S_{m}, \mathbb{Z}\right)=\mathbb{Z}^{m+1}$. In contrast, no example is known of a finitely presented group that is not large but has $\operatorname{vb}_{1}(G)=\infty$ (see [Button 2010; Lackenby 2010]). Since amenable groups do not contain nonabelian free subgroups, one might hope to resolve this issue by finding a finitely presented amenable group with $\operatorname{vb}_{1}(G)=\infty$, but this seems to be a nontrivial matter.

We shall prove in this paper that for large classes of finitely presented soluble groups $\operatorname{vb}_{1}(G)$ is always finite. One would like to prove that the same is true for all finitely presented soluble groups, but here one faces the profound difficulty of deciding which soluble groups admit finite presentations; this is unknown even for abelian-by-polycyclic and nilpotent-by-abelian groups.

In the case of metabelian groups, finite presentability is completely understood in terms of the Bieri-Strebel invariant [Bieri and Strebel 1980]. Some sufficient conditions for finite presentability of nilpotent-by-abelian groups were considered by McIsaac [1984] and later Groves [1991]. In the case of $S$-arithmetic nilpotentby-abelian groups $G$ one knows more thanks to the work of Abels [1987]: if $G$ is an extension of a nilpotent group $N$ by an abelian group $Q$ then $G$ is finitely

MSC2010: 20F16, 20J05.

Keywords: soluble groups, virtual first betti number, nilpotent-by-abelian-by-finite. 
presented if and only if it is of type $\mathrm{FP}_{2}$, which it is if and only if $H_{2}(N, \mathbb{Z})$ is finitely generated as a $\mathbb{Z} Q$-module (where the $Q$ action is induced by conjugation) and $^{1} G / N^{\prime}$ is finitely presented as a group. The first of these conditions is an easy consequence of the fact that $\mathbb{Z} Q$ is a Noetherian ring, and the second is a corollary of a result in [Bieri and Strebel 1980] that every metabelian quotient of a group of type $\mathrm{FP}_{2}$ that does not contain noncyclic free subgroups is finitely presented. The case where $G$ is an extension of an abelian normal subgroup $A$ by a polycyclic group $Q$ was approached by Brookes and Groves who studied modules over crossed products of a division ring by a free abelian group; see [Brookes and Groves 1995; 2000; 2002].

Given this background, the natural place to begin our investigation into the virtual first betti number of finitely presented soluble groups is in the setting of metabelian groups. Using methods from commutative algebra, we prove (Theorem 4.3) that if $G$ is finitely presented and metabelian, then $\operatorname{vb}_{1}(G)$ is finite. (The hypothesis that one actually needs to impose on $G$ is somewhat weaker than finite presentability; see Remark 6.5.) The metabelian case is used in the proof of our main theorem, which is the following.

Theorem A. Let $G$ be a finitely presented group. If $G$ is nilpotent-by-abelian-byfinite, then $\mathrm{vb}_{1}(G)$ is finite.

Our proof of this theorem relies on the fact that all metabelian quotients of soluble groups of type $\mathrm{FP}_{2}$ are finitely presented [Bieri and Strebel 1980, Theorem 5.5], as well as a technical result concerning the homology of subgroups of finite index (Proposition 6.2). Groves, Kochloukova and Rodrigues [Groves et al. 2008, Theorem A] proved that if an abelian-by-polycyclic group $G$ is of type $\mathrm{FP}_{3}$ then it is nilpotent-by-abelian-by-finite, in which case $\mathrm{vb}_{1}(G)$ is finite by Theorem A. The same is true of all soluble groups of type $\mathrm{FP}_{\infty}$, because they are constructible [Kropholler 1986], hence nilpotent-by-abelian-by-finite, but in this case stronger finiteness results were already known: constructible soluble groups are obtained from the trivial group by finite sequences of ascending HNN extensions and finite extensions, from which it follows that they have finite Prüfer rank (i.e., there is an upper bound on the number of generators for the finitely generated subgroups).

It is natural to wonder if Theorem A might remain true when the field of rationals $\mathbb{Q}$ in the definition of virtual betti number is replaced with other coefficient fields, such as the field with $p$ elements $F_{p}$. We shall see in Section 5 that it does not.

Conjecture. If $G$ is finitely presented and soluble, then $\operatorname{vb}_{1}(G)$ is finite.

It is difficult to construct finitely presented soluble groups that are not nilpotentby-abelian-by-finite. The examples provided by the constructions of Robinson and Strebel [1982] all satisfy the conjecture.

\footnotetext{
${ }^{1}$ Throughout this article, $H^{\prime}$ denotes the commutator subgroup of a group $H$.
} 
While editing the final version of this work, we learnt that Andrei Jaikin-Zapirain has, in unpublished work, also proved Theorem A in the metabelian case. Higher dimensional analogues of Theorem A are considered in the forthcoming $\mathrm{PhD}$ thesis of Fatemeh Mokari.

\section{Preliminary results}

2A. Preliminaries on finitely presented metabelian groups. We fix a short exact sequence of groups $A \longmapsto G \rightarrow Q$, where $A$ and $Q$ are abelian and $G$ is finitely generated. The action of $G$ on $A$ by conjugation induces an action of $Q$, which enables us to regard $A$ as a right $\mathbb{Z} Q$-module. Because $G$ is finitely generated and $Q$ is finitely presented, $A$ is finitely generated as a $\mathbb{Z} Q$-module.

Associated to a nonzero real character $\chi: Q \rightarrow \mathbb{R}$ one has the monoid

$$
Q_{\chi}=\{g \in Q \mid \chi(g) \geq 0\} .
$$

The character sphere $S(Q)$ is the set of equivalence classes in $\operatorname{Hom}(Q, \mathbb{R}) \backslash\{0\}$ under the relation that identifies $\chi_{1} \sim \chi_{2}$ if $\chi_{1}=\lambda \chi_{2}$ for some $\lambda>0$. We write $[\chi]$ for the class of $\chi$. Following [Bieri and Strebel 1980], let

$$
\Sigma_{A}(Q)=\left\{[\chi] \mid A \text { is finitely generated as a } \mathbb{Z} Q_{\chi} \text {-module }\right\} .
$$

By definition, the $\mathbb{Z} Q$-module $A$ is 2-tame if $\Sigma_{A}(Q)^{c}=S(Q) \backslash \Sigma_{A}(Q)$ contains no pair of antipodal points. According to [op. cit., Theorem 5.4], $G$ is finitely presented if and only if $A$ is a 2 -tame $\mathbb{Z} Q$-module, and this happens precisely when $G$ is of homological type $\mathrm{FP}_{2}$. We refer the reader to [Bieri 1981] for general results concerning groups of type $\mathrm{FP}_{m}$. If $A_{1} \longmapsto A_{2} \rightarrow A_{3}$ is an exact sequence of finitely generated $\mathbb{Z} Q$-modules, then $\Sigma_{A_{2}}(Q)^{c}=\Sigma_{A_{1}}(Q)^{c} \cup \Sigma_{A_{3}}(Q)^{c}$ (see [Bieri and Strebel 1980, Proposition 2.2]), hence every quotient of a 2 -tame $\mathbb{Z} Q$-module is 2-tame.

2B. Tensor products and finite presentability. Let $R$ be a noetherian commutative ring with unit 1 and let $W$ be a finitely generated $R Q$-module. As above, we have a Sigma invariant $\Sigma_{W}(Q)=\left\{[\chi] \mid W\right.$ is finitely generated as an $R Q_{\chi}$-module $\}$, and $W$ is defined to be 2-tame as an $R Q$-module if $\Sigma_{W}^{c}(Q)=S(Q) \backslash \Sigma_{W}(Q)$ has no pair of antipodal points.

The question of when the tensor square $W \otimes_{R} W$ is finitely generated as an $R Q$-module (with $Q$ acting diagonally) is addressed in [Bieri and Groves 1985], where it is shown that $[\chi]$ lies in $\Sigma_{W}^{c}(Q)$ if and only if the ring $S=R Q / \operatorname{ann}_{R Q}(W)$ admits a real valuation $v: S \rightarrow \mathbb{R} \cup\{\infty\}$ (in the sense of Bourbaki) that extends $\chi$ and is such that the restriction $v_{0}$ of $v$ to the image $\bar{R}$ of $R$ in $S$ is nonnegative and discrete. By [loc. cit.], $W \otimes_{R} W$ is finitely generated as an $R Q$-module if and only if there is no pair of antipodal elements $[\chi],[-\chi] \in \Sigma_{W}^{c}(Q)$ that can be 
lifted to valuations of $S$ that have the same restriction $v_{0}$ to $\bar{R}$, with $v_{0}$ discrete and nonnegative. (These last conditions on $v_{0}$ are automatic if $\bar{R}$ is $\mathbb{Z}$.)

Returning to the context of Section 2A, we apply these general considerations with $W=A \otimes \mathbb{Q}$ and $R=\mathbb{Q}$, in which case $W \otimes_{R} W \cong\left(A \otimes_{\mathbb{Z}} A\right) \otimes_{\mathbb{Z}} \mathbb{Q}$. We deduce that if there exists a group extension $A \longmapsto G \rightarrow Q$, with $G$ finitely presented, then $W=A \otimes \mathbb{Q}$ is 2-tame as a $\mathbb{Q} Q$-module, and $W \otimes_{R} W \cong\left(A \otimes_{\mathbb{Z}} A\right) \otimes_{\mathbb{Z}} \mathbb{Q}$ is finitely generated as a $\mathbb{Q} Q$-module via the diagonal $Q$-action.

We shall also need a refinement of this observation that involves the annihilator $\operatorname{ann}_{\mathbb{Z} Q}(A)$ of $A$ in $\mathbb{Z} Q$, which we denote $I$. Bieri and Strebel [1981, (1.3)] prove that

$$
\Sigma_{A}(Q)=\Sigma_{\mathbb{Z} Q / I}(Q) .
$$

Thus if $A$ is 2 -tame as a $\mathbb{Z} Q$-module, then so is $\mathbb{Z} Q / I$.

Lemma 2.1. If there exists a group extension $A \longmapsto G \rightarrow Q$ with $A$ and $Q$ abelian and $G$ finitely presented, and $I=\operatorname{ann}_{\mathbb{Z} Q}(A)$, then $(\mathbb{Z} Q / I) \otimes_{\mathbb{Z}}(\mathbb{Z} Q / I) \otimes_{\mathbb{Z}} \mathbb{Q}$ is finitely generated as a $\mathbb{Q} Q$-module via the diagonal $Q$-action.

2C. Preliminaries on commutative algebra. We will need the following basic facts from commutative algebra; for details see, for example, [Bourbaki 1961-1965; Atiyah and Macdonald 1969; Eisenbud 1995]. Let $Q$ be a finitely generated abelian group and recall that the Krull dimension of a commutative ring is the supremum of the lengths of all chains of prime ideals in the ring.

(1) The radical $\sqrt{J}$ of each ideal $J \triangleleft \mathbb{Q} Q$ is the intersection of the finitely many prime ideals that contain $J$ and are minimal subject to this condition.

(2) Finite dimensional $\mathbb{Q}$-algebras are Artinian and thus have Krull dimension 0.

Throughout, if $R$ is a commutative ring and $m$ a positive integer, then $R^{m}$ will denote the subring generated by $m$-th powers, except that $\mathbb{Z}^{n}$ and $\mathbb{Q}^{n}$ will denote Cartesian powers. Where no ring is specified, tensor products are assumed to be taken over $\mathbb{Z}$.

\section{A finiteness result in commutative algebra}

Lemma 2.1 assures us that the following theorem applies to the modules that arise from short exact sequences $N \longmapsto G \rightarrow \mathbb{Z}^{n}$ associated to finitely presented metabelian groups.

Theorem 3.1. Let $Q \cong \mathbb{Z}^{n}$ be a group and let $S=\mathbb{Z} Q / I$ be a commutative ring such that $\left(S \otimes_{\mathbb{Z}} S\right) \otimes_{\mathbb{Z}} \mathbb{Q}$ is finitely generated as a $\mathbb{Q} Q$-module via the diagonal $Q$-action. Then,

$$
\sup _{m} \operatorname{dim}_{\mathbb{Q}}\left(S \otimes_{\mathbb{Z} Q^{m}} \mathbb{Q}\right)<\infty
$$


Proof. Let $B=S \otimes \mathbb{Q}=\mathbb{Q} Q / J$ and for each positive integer $m$ define $J_{m} \triangleleft \mathbb{Q}$ to be $\left(J, Q^{m}-1\right)$ and

$$
B_{m}:=B \otimes \mathbb{Q} Q^{m} \mathbb{Q}=\mathbb{Q} Q / J_{m} \cong S \otimes_{\mathbb{Z} Q^{m}} \mathbb{Q} .
$$

As $\mathbb{Q} Q /\left(Q^{m}-1\right)$ is finite dimensional over $\mathbb{Q}$, so is $B_{m}=\mathbb{Q} Q / J_{m}$. Hence $B_{m}$ has Krull dimension 0; i.e., every prime ideal in $B_{m}$ is a maximal one. Therefore, the finite collection of primes ideals $P_{m, t}$ whose intersection is $\sqrt{B_{m}}$ are the only prime ideals in $\mathbb{Q} Q$ above $J_{m}$, and each of the quotients $\mathbb{Q} Q / P_{m, t}$ is a field.

We shall establish the theorem by proving the following:

Claim 1. There exist only finitely many fields $F$ such that for some $m \geq 1$ (depending on $F$ ) the field $F$ is a quotient of $B_{m}$.

Claim 1 provides an integer $m_{0}$ such that if a field $F$ is a quotient of $B_{m}$ then the natural map $\mathbb{Q} Q \rightarrow F$ factors through $\mathbb{Q} Q /\left(Q^{m_{0}}-1\right)$.

Claim 2. If $m_{0}$ divides $m$ then $J_{m}=J_{m r}$ for every $r \in \mathbb{N}$.

To see that the theorem follows from these claims, note that for an arbitrary positive integer $m$ we have $J_{m} \supseteq J_{m m_{0}}=J_{m_{0}}$, whence

$$
\begin{aligned}
\operatorname{dim}_{\mathbb{Q}}\left(\mathbb{Q} Q / J_{m}\right) & \leq \operatorname{dim}_{\mathbb{Q}}\left(\mathbb{Q} Q / J_{m_{0}}\right) \leq \operatorname{dim}_{\mathbb{Q}}\left(\mathbb{Q} Q /\left(Q^{m_{0}}-1\right)\right) \\
& =\operatorname{dim}_{\mathbb{Q}} \mathbb{Q}\left[Q / Q^{m_{0}}\right]=m_{0}^{n} .
\end{aligned}
$$

Proof of Claim 1. Our hypothesis on $S$ implies that $B \otimes_{\mathbb{Q}} B$ is finitely generated as $\mathbb{Q} Q$-module via the diagonal $Q$-action, by $d$ elements say. Let $F$ be a field quotient of $B_{m}$ and let $\theta: \mathbb{Q} Q \rightarrow F$ be the canonical projection; so $Q^{m}-1 \subseteq \operatorname{ker}(\theta)$. Then, $\theta(Q)$ is a finitely generated multiplicative subgroup of $F^{*}$ that has finite exponent and $F$, being finite dimensional over $\mathbb{Q}$, embeds in $\mathbb{C}$. Hence $\theta(Q)$ is a finite cyclic group, generated by a root of unity, $\epsilon$ of order $s$, say. Thus we obtain a subgroup $H<Q$ such that $Q / H$ is cyclic of order $s$ and $H-1 \subseteq \operatorname{ker}(\theta)$. Now, $F \cong \mathbb{Q}[x] /(f)$, where $f$ is the minimal polynomial of $\epsilon$ over $\mathbb{Q}$. And $f$ is an irreducible factor of $x^{s}-1$ in $\mathbb{Q}[x]$, whose zeroes are distinct roots of unity with order precisely $s$. Thus $\operatorname{dim}_{\mathbb{Q}} F=\operatorname{deg}(f)=\varphi(s)$, where $\varphi$ is Euler's totient function. On the other hand, $F \otimes_{\mathbb{Q}} F$ is an epimorphic image of the $\mathbb{Q} Q$-module $B \otimes_{\mathbb{Q}} B$ and the action of $Q$ on $F \otimes_{\mathbb{Q}} F$ factors through the action of $Q / H$, so $F \otimes_{\mathbb{Q}} F$ is generated as a $\mathbb{Q}[Q / H]$-module by $d$ elements. Hence

$$
\varphi(s)^{2}=\left(\operatorname{dim}_{\mathbb{Q}} F\right)^{2}=\operatorname{dim}_{\mathbb{Q}}\left(F \otimes_{\mathbb{Q}} F\right) \leq d \operatorname{dim}_{\mathbb{Q}} \mathbb{Q}[Q / H]=d s .
$$

An elementary calculation shows that $\varphi(n) / \sqrt{n} \rightarrow \infty$ as $n \rightarrow \infty$, so for fixed $d$ there are only finitely many possible values of $s$ and $\epsilon$. Let $b$ be a natural number such that the order of $\epsilon$ is at most $b$. Then, the order of $\epsilon$ is a divisor of $m_{0}=b$ ! and 
Since $\mathbb{Q} Q /\left(Q^{m_{0}}-1\right)$ is finite dimensional over $\mathbb{Q}$ it has Krull dimension 0 , so has only finitely many prime ideals and finitely many field quotients. This completes the proof of Claim 1.

Proof of Claim 2. Since $m_{0}$ divides $m$ we have $J_{m} \subseteq J_{m_{0}}$, so the prime ideals containing $J_{m_{0}}$ also contain $J_{m}$. On the other hand, we saw earlier that for each of the prime ideals $P_{m, i}$ containing $J_{m}$, the quotient $F_{i}:=\mathbb{Q} Q / P_{m, i}$ is a field. By definition, $m_{0}$ is such that $\mathbb{Q} Q \rightarrow F_{i}$ factors through $\mathbb{Q} Q /\left(Q^{m_{0}}-1\right)$, and therefore $P_{m, i}$ (which already contains $J \subset J_{m}$ ) contains $J_{m_{0}}=\left(J, Q^{m_{0}}-1\right)$. The radical of $J_{m}$ is the intersection of the prime ideals containing it, so

$$
\sqrt{J_{m}}=\sqrt{J_{m_{0}}} .
$$

Arguing by induction on $r$, Claim 2 will follow if we can prove that for every prime number $p$ we have $J_{m}=J_{m p}$, which is equivalent to the assertion that $q^{m}-1 \in J_{m p}$ for all $q \in Q$.

We now fix $q \in Q$. From the preceding argument, $\sqrt{J_{m}}=\sqrt{J_{m p}}$. In particular, $Q^{m}-1 \subseteq J_{m} \subseteq \sqrt{J_{m}}=\sqrt{J_{m p}}$, so there is a natural number $s$ (over which we have no control) such that

$$
\left(q^{m}-1\right)^{s} \in J_{m p} .
$$

As $Q^{m p}-1 \subseteq J_{m p}$, we also have

$$
q^{m p}-1 \in J_{m p}
$$

Let $g(x)$ be the greatest common divisor of $x^{p m}-1$ and $\left(x^{m}-1\right)^{s}$ in $\mathbb{Q}[x]$. In characteristic zero, the polynomial $x^{p m}-1$ has no repeated roots, so neither does $g(x)$. Since $g(x)$ divides $\left(x^{m}-1\right)^{s}$, it must actually divide $x^{m}-1$, so in fact $g(x)=x^{m}-1$. From (3-1), (3-2) and Bézout's lemma, we have $g(q) \in J_{p m}$. Since $q \in Q$ is arbitrary, this implies that $J_{m p}=J_{m}$.

\section{The main theorem for metabelian groups}

In this section we prove that all finitely presented metabelian groups have finite virtual first betti number. The proof relies on the finiteness theorem proved in the previous section and two technical lemmas, the first of which is a simple observation about commensurable groups.

Lemma 4.1. Let $G$ be a group. If $G_{0}<G$ is a subgroup of finite index, then $\operatorname{vb}_{1}(G)=\operatorname{vb}_{1}\left(G_{0}\right)$.

Proof. By definition, $\operatorname{vb}_{1}(G)=\sup _{M} \operatorname{dim} H_{1}(M, \mathbb{Q})$, where the supremum is taken over finite-index subgroups of $G$. If $M$ has finite index in $G_{0}$, then it also has finite index in $G$, so $\operatorname{vb}_{1}(G) \geq \operatorname{vb}_{1}\left(G_{0}\right)$. Conversely, if $S$ has finite index in $G$, then 
$S_{0}=G_{0} \cap S$ has finite index in $G_{0}$, and since it also has finite index in $S$, we have $\operatorname{dim} H_{1}\left(S_{0}, \mathbb{Q}\right) \geq \operatorname{dim} H_{1}(S, \mathbb{Q})$, so $\operatorname{vb}_{1}\left(G_{0}\right) \geq \operatorname{vb}_{1}(G)$.

Lemma 4.2. Let $A \longmapsto G \rightarrow Q$ be a short exact sequence of groups with $A$ and $Q$ abelian and let $n$ be the torsion-free rank of $Q$. Then:

(a) Writing $[G, A]=\left\langle\left\{[g, a]=g^{-1} a^{-1} g a \mid g \in G, a \in A\right\}\right\rangle$, we have

$$
\operatorname{dim}_{\mathbb{Q}} H_{1}(G, \mathbb{Q}) \leq \operatorname{dim}_{\mathbb{Q}}(A /[G, A] \otimes \mathbb{Q})+n .
$$

In the split case, $G=A \rtimes Q$, we have $H_{1}(G, \mathbb{Q}) \cong(G /[G, A]) \otimes_{\mathbb{Z}} \mathbb{Q}$, and

$$
\operatorname{dim}_{\mathbb{Q}} H_{1}(G, \mathbb{Q})=\operatorname{dim}_{\mathbb{Q}}(A /[G, A] \otimes \mathbb{Q})+n .
$$

(b) If $G_{m}$ is a subgroup of finite index in $G$ and $Q_{m}$ is the image of $G_{m}$ in $Q$, then

$$
\operatorname{dim}_{\mathbb{Q}} H_{1}\left(G_{m}, \mathbb{Q}\right) \leq \operatorname{dim}_{\mathbb{Q}}\left(A \otimes_{\mathbb{Z} Q_{m}} \mathbb{Q}\right)+n .
$$

In the split case, $G_{m}=\left(A \cap G_{m}\right) \rtimes Q_{m}$, equality is attained:

$$
\operatorname{dim}_{\mathbb{Q}} H_{1}\left(G_{m}, \mathbb{Q}\right)=\operatorname{dim}_{\mathbb{Q}}\left(A \otimes_{\mathbb{Z} Q_{m}} \mathbb{Q}\right)+n .
$$

(c) If $G=A \rtimes Q$ and $\mathscr{B}$ denotes the set of subgroups of finite index in $Q$, then

$$
\operatorname{vb}_{1}(G)=\sup _{S \in \mathscr{P}} \operatorname{dim}_{\mathbb{Q}}\left(A \otimes_{\mathbb{Z} S} \mathbb{Q}\right)+n .
$$

Proof. (a) As $[G, A] \subseteq[G, G]$, we see that $H_{1}(G, \mathbb{Z})=G /[G, G]$ is a quotient of $G /[G, A]$. So from the central extension $A /[G, A] \longmapsto G /[G, A] \rightarrow Q$, we get $\operatorname{dim}_{\mathbb{Q}} H_{1}(G, \mathbb{Q}) \leq \operatorname{dim}_{\mathbb{Q}}(A /[G, A] \otimes \mathbb{Q})+\operatorname{dim}_{\mathbb{Q}}(Q \otimes \mathbb{Q})=\operatorname{dim}_{\mathbb{Q}}(A /[G, A] \otimes \mathbb{Q})+n$.

If $G=A \rtimes Q$ then, using that $A, Q$ are abelian and $A$ is normal in $G$, we get $[G, G]=[A Q, A Q]=[Q, A] \subseteq[G, A] \subseteq[G, G]$, hence $[G, G]=[G, A]$ and $A /[G, A] \longmapsto G /[G, G] \rightarrow Q$ is an exact sequence of abelian groups.

(b) We consider the short exact sequence $A_{m} \longmapsto G_{m} \rightarrow Q_{m}$, where $A_{m}=A \cap G_{m}$. From part (a) we have

$$
\operatorname{dim}_{\mathbb{Q}} H_{1}\left(G_{m}, \mathbb{Q}\right) \leq \operatorname{dim}_{\mathbb{Q}}\left(A_{m} \otimes_{\mathbb{Z} Q_{m}} \mathbb{Q}\right)+n,
$$

with equality if the sequence splits. Furthermore, since $A / A_{m}$ is finite we have

$$
0=\operatorname{Tor}_{1}^{\mathbb{Z} Q_{m}}\left(A / A_{m}, \mathbb{Q}\right) \quad \text { and }\left(A / A_{m}\right) \otimes_{\mathbb{Z} Q_{m}} \mathbb{Q}=0 .
$$

Thus there is an exact sequence (part of the long exact sequence in Tor associated to $\left.A \cap G_{m} \longmapsto A \rightarrow A /\left(A \cap G_{m}\right)\right)$

$$
0=\operatorname{Tor}_{1}^{\mathbb{Z} Q_{m}}\left(A / A_{m}, \mathbb{Q}\right) \rightarrow A_{m} \otimes_{\mathbb{Z} Q_{m}} \mathbb{Q} \rightarrow A \otimes_{\mathbb{Z} Q_{m}} \mathbb{Q} \rightarrow\left(A / A_{m}\right) \otimes_{\mathbb{Z} Q_{m}} \mathbb{Q}=0,
$$

whence $A_{m} \otimes_{\mathbb{Z} Q_{m}} \mathbb{Q} \cong A \otimes_{\mathbb{Z} Q_{m}} \mathbb{Q}$. Thus, we may replace $A_{m} \otimes_{\mathbb{Z} Q_{m}} \mathbb{Q}$ in (4-1) by $A \otimes_{\mathbb{Z} Q_{m}} \mathbb{Q}$, and (b) is proved. 
(c) From the first part of (b) we have

$$
\operatorname{vb}_{1}(G) \leq \sup _{S \in \mathscr{P}} \operatorname{dim}_{\mathbb{Q}}\left(A \otimes_{\mathbb{Z} S} \mathbb{Q}\right)+n,
$$

and to obtain the reverse inequality, we use the second part of (b)

$$
\sup _{S \in \mathscr{P}} \operatorname{dim}_{\mathbb{Q}}\left(A \otimes_{\mathbb{Z} S} \mathbb{Q}\right)+n=\sup _{S \in \mathscr{R}} \operatorname{dim}_{\mathbb{Q}} H_{1}(A \rtimes S, \mathbb{Q}),
$$

noting that $A \rtimes S$ has finite index in $G$.

Theorem 4.3. Let $A \longmapsto G \rightarrow Q$ be a short exact sequence of groups with $A$ and $Q$ abelian. If $G$ is finitely presented then its virtual first betti number $\mathrm{vb}_{1}(G)$ is finite.

Proof. By passing to a subgroup of finite index in $Q$ and replacing $G$ by the inverse image of this subgroup, we may assume that $Q$ is free abelian. Lemma 4.1 assures us that it is enough to consider this case, and Lemma 4.2(b) tells us that we will be done if we can establish an upper bound on $\operatorname{dim}_{\mathbb{Q}}\left(A \otimes_{\mathbb{Z} Q_{m}} \mathbb{Q}\right)$ as $Q_{m}$ ranges over the subgroups of finite index in $Q$.

Recall that $A$ is finitely generated as a $\mathbb{Z} Q$-module, say by $d$ elements. Thus, denoting the annihilator $\operatorname{ann}_{\mathbb{Z} Q}(A)=\{\lambda \in \mathbb{Z} Q \mid A \lambda=0\}$ by $I$, we have an epimorphism of $\mathbb{Z} Q$-modules

$$
(\mathbb{Z} Q / I)^{[d]}=\mathbb{Z} Q / I \oplus \cdots \oplus \mathbb{Z} Q / I \rightarrow A
$$

that induces an epimorphism of $\mathbb{Q}$-vector spaces

$$
\left((\mathbb{Z} Q / I) \otimes_{\mathbb{Z} Q_{m}} \mathbb{Q}\right)^{[d]}=(\mathbb{Z} Q / I)^{[d]} \otimes_{\mathbb{Z} Q_{m}} \mathbb{Q} \rightarrow A \otimes_{\mathbb{Z} Q_{m}} \mathbb{Q} .
$$

Thus,

$$
\operatorname{dim}_{\mathbb{Q}}\left(A \otimes_{\mathbb{Z} Q_{m}} \mathbb{Q}\right) \leq d \operatorname{dim}_{\mathbb{Q}}\left((\mathbb{Z} Q / I) \otimes_{\mathbb{Z} Q_{m}} \mathbb{Q}\right)
$$

and it suffices to show that

$$
\sup _{m} \operatorname{dim}_{\mathbb{Q}}\left((\mathbb{Z} Q / I) \otimes_{\mathbb{Z} Q_{m}} \mathbb{Q}\right)<\infty .
$$

For every $m$ there is a natural number $\alpha_{m}$ such that $Q^{\alpha_{m}} \subseteq Q_{m}$, and $\mathbb{Z} Q / I \otimes_{\mathbb{Z} Q_{m}} \mathbb{Q}$ is a quotient of $\mathbb{Z} Q / I \otimes_{\mathbb{Z} Q^{\alpha_{m}}} \mathbb{Q}$. Thus,

$$
\operatorname{dim}_{\mathbb{Q}}\left((\mathbb{Z} Q / I) \otimes_{\mathbb{Z} Q_{m}} \mathbb{Q}\right) \leq \operatorname{dim}_{\mathbb{Q}}\left((\mathbb{Z} Q / I) \otimes_{\mathbb{Z} Q^{\alpha_{m}}} \mathbb{Q}\right),
$$

and we have reduced to showing that

$$
\sup _{s} \operatorname{dim}_{\mathbb{Q}}\left((\mathbb{Z} Q / I) \otimes_{\mathbb{Z} Q^{s}} \mathbb{Q}\right)<\infty .
$$

The theorem now follows from Lemma 2.1 and Theorem 3.1. 


\section{Characteristic $p$ case}

In this section we shall construct examples which show that the restriction to fields of characteristic 0 in Theorem A is essential, even in the metabelian case. ${ }^{2}$ To this end, we consider the mod $p$ virtual first betti number of a finitely generated group $G$,

$$
\operatorname{vb}_{1}^{(p)}(G)=\sup \left\{\operatorname{dim} H_{1}\left(S, F_{p}\right) \mid S<G \text { of finite index }\right\} .
$$

Proposition 5.1. For every prime $p$ there exist finitely presented metabelian groups $\Gamma$ such that $\mathrm{vb}_{1}{ }^{(p)}(\Gamma)$ is infinite.

Proof. Let $Q$ be a free abelian group with generators $x$ and $y$ and let $A=F_{p} Q / I$, where $I$ is the ideal of $F_{p} Q$ generated by $y-x^{2}+x-1$. Then,

Consider

$$
A \cong F_{p}\left[x, x^{-1}, \frac{1}{x^{2}-x+1}\right] .
$$

$$
A_{m}=A \otimes_{\mathbb{Z} Q^{p^{m}}} F_{p} \cong F_{p} Q /\left(I, Q^{p^{m}}-1\right) .
$$

Since $\left(x^{2}-x+1\right)^{p^{m}}-1=x^{2 p^{m}}-x^{p^{m}}+1-1=x^{p^{m}}\left(x^{p^{m}}-1\right)$, we have

$$
\begin{aligned}
A_{m} & =F_{p}\left[x, x^{-1}, \frac{1}{x^{2}-x+1}\right] /\left(x^{p^{m}}-1,\left(x^{2}-x+1\right)^{p^{m}}-1\right) \\
& =F_{p}\left[x, x^{-1}, \frac{1}{x^{2}-x+1}\right] /\left(x^{p^{m}}-1\right)
\end{aligned}
$$

is the localisation

$$
B_{m} S^{-1}
$$

where $B_{m}=F_{p}\left[x, x^{-1}\right] /\left(x^{p^{m}}-1\right)$ and $S$ is the image of $\left\{\left(x^{2}-x+1\right)^{j}\right\}_{j \geq 1}$ in $B_{m}$. Note that $x^{p^{m}}-1$ and $x^{2}-x+1$ do not have a common root in any finite field extension of $F_{p}$, for if $z$ were a common root we would have $1=z^{2 p^{m}}=(z-1)^{p^{m}}=$ $z^{p^{m}}-1=0$, which is a contradiction. Thus the polynomials $x^{p^{m}}-1$ and $\left(x^{2}-x+1\right)^{j}$ are coprime in $F_{p}\left[x, x^{-1}\right]$; i.e., they generate the whole ring as an ideal, and so the elements of $S$ are invertible in $B_{m}$. Therefore $B_{m} S^{-1}=B_{m}$ and

$$
\operatorname{dim}_{F_{p}} A_{m}=\operatorname{dim}_{F_{p}} B_{m} S^{-1}=\operatorname{dim}_{F_{p}} B_{m}=p^{m} .
$$

Now define

$$
\Gamma=A \rtimes Q \quad \text { and } \quad \Gamma_{m}=A \rtimes Q^{p^{m}} .
$$

Then, as in the split case of Lemma 4.2(b) (with coefficients in $F_{p}$ in place of $\mathbb{Q}$ ),

$$
\operatorname{dim}_{F_{p}} H_{1}\left(\Gamma_{m}, F_{p}\right)=\operatorname{dim}_{F_{p}} A_{m}+2=p^{m}+2,
$$

${ }^{2}$ John Wilson [1998] proved that the dimension of $H_{1}\left(S, F_{p}\right)$ can grow at most like the square root of the index $[G: S]$. Jack Button [2010] exhibited a finitely presented soluble group that exhibits this growth for all $p$. 
which tends to infinity with $m$.

By the calculation [Bieri and Strebel 1981, Theorem 5.2] of $\Sigma_{A}(Q)$ for $A=$ $F_{p} Q / I$, where the ideal $I$ is 1 -generated, or by the link between $\Sigma_{A}^{c}(Q)$ and valuation theory (as described in Section 2B), we have

$$
\Sigma_{A}^{c}(Q)=\left\{\left[\chi_{1}\right],\left[\chi_{2}\right],\left[\chi_{3}\right]\right\},
$$

with

$$
\begin{array}{lll}
\chi_{1}(x)=0, & \chi_{2}(x)=1, & \chi_{3}(x)=-1, \\
\chi_{1}(y)=1, & \chi_{2}(y)=0, & \chi_{3}(y)=-2 .
\end{array}
$$

Thus, $A$ is 2 -tame as a $\mathbb{Z} Q$-module, and by the classification of finitely presented metabelian groups in [Bieri and Strebel 1980], $\Gamma$ is finitely presented.

Corollary 5.2. There exists a finitely presented metabelian group $G$ such that for the class $\mathscr{A}$ of all subgroups of finite index in $G$,

$$
\sup _{M \in \mathscr{A}} d(M)=\infty
$$

where $d(M)$ is the minimal number of generators of $M$.

Proof. Immediate, since $d(M) \geq \operatorname{dim}_{F_{p}} H_{1}\left(M, F_{p}\right)$.

It is natural to wonder if the lack of finiteness exhibited in the preceding proposition might be avoided by restricting to subgroups whose index is coprime to $p$. The following refinement shows that this is not the case.

Proposition 5.3. Let $p$ be a prime. There exist finitely presented metabelian groups $G$ such that

$$
\sup \left\{\operatorname{dim}_{F_{p}} H_{1}\left(S, F_{p}\right) \mid S \in \mathscr{A}_{p}\right\}=\infty
$$

where

$$
\mathscr{A}_{p}=\{S \leq G \mid[G: S] \text { is finite and coprime to } p\} .
$$

Proof. Let $A=F_{p}\left[x, x^{-1},(x+1)^{-1}\right]$ and let $Q$ be a free abelian group of rank 2 whose generators $x_{1}, x_{2}$ act on $A$ as multiplication by $x$ and $x+1$, respectively. We consider the group $G=A \rtimes Q$. As an $F_{p}[Q]$-module, $A \cong F_{p}[Q] / I$ where $I$ is the ideal generated by $x_{2}-x_{1}-1$, and the argument given in the preceding proposition shows that $\Sigma_{A}(Q)^{c}$ consists of precisely 3 points, no pair of which is antipodal. Therefore, $G$ is finitely presented.

Let $F$ be a finite field with $p^{r}$ elements, $r \geq 2$. Let $w$ be a generator of the multiplicative group $F^{*}=F \backslash\{0\}$. Let $Q_{r}$ be the kernel of the homomorphism $Q \rightarrow F^{*}$ defined by $x_{1} \mapsto w$ and $x_{2} \mapsto w+1$. Let $G_{r}=A \rtimes Q_{r}$ and note that $\left|G / G_{r}\right|=\left|Q / Q_{r}\right|=p^{r}-1$ is coprime to $p$.

The ring epimorphism $A \rightarrow F$ sending $x$ to $w$ provides an epimorphism of the underlying additive groups which extends to a group epimorphism $A \rtimes Q_{r} \rightarrow F \times \mathbb{Z}^{2}$. 
Since $\operatorname{dim}_{F_{p}} F=r$, it follows that $\operatorname{dim}_{F_{p}} H_{1}\left(G_{r}, F_{p}\right) \geq r+2$. And, $r \geq 2$ was arbitrary.

\section{Beyond the metabelian case}

In this section we shall prove Theorem A, but first we present a consequence of Theorem 4.3 that describes what one can deduce about towers of finite-index subgroups above the commutator subgroup in amenable and related groups.

Proposition 6.1. Let $G$ be a group of type $\mathrm{FP}_{2}$ that does not contain a nonabelian free group and let $\mathscr{C}$ be the set of finite-index subgroups in $G$ that contain the commutator subgroup $G^{\prime}$. Then, $\sup _{M \in \mathscr{C}} \operatorname{dim}_{\mathbb{Q}} H_{1}(M, \mathbb{Q})<\infty$.

Proof. By [Bieri and Strebel 1980, Theorem 5.5], $G / G^{\prime \prime}$ is finitely presented. Since $M \supseteq G^{\prime}$, we have $M^{\prime} \supseteq G^{\prime \prime}$ and can replace $G$ by $G / G^{\prime \prime}$ and $M$ by $M G^{\prime \prime} / G^{\prime \prime}$ without changing $H_{1}(M, \mathbb{Q})$. Then we can apply Theorem 4.3.

Our proof of Theorem A relies on the following proposition, which is of interest in its own right.

Proposition 6.2. Let $N \longmapsto G \rightarrow Q$ be a short exact sequence of groups, where $N$ is nilpotent, $Q$ is abelian and $G$ is finitely generated. Let $G_{n}$ be a subgroup of finite index in $G$ and let $\bar{G}_{n}$ be the image of $G_{n}$ in the metabelian group $G / N^{\prime}$. Then,

$$
\operatorname{dim}_{\mathbb{Q}} H_{1}\left(G_{n}, \mathbb{Q}\right)=\operatorname{dim}_{\mathbb{Q}} H_{1}\left(\bar{G}_{n}, \mathbb{Q}\right) .
$$

Proof. We argue using the Malcev completion $j_{N}: N \rightarrow N^{*}$ [Malcev 1949]. According to [Quillen 1969, Appendix A, Corollary 3.8], for any nilpotent group $N$, the homomorphism $j_{N}: N \rightarrow N^{*}$ is characterized up to isomorphism by the following properties:

(a) $N^{*}$ is nilpotent and uniquely divisible.

(b) ker $j_{N}$ is the torsion subgroup of $N$.

(c) For every $x \in N^{*}$, there is a positive integer $n$ such that $x^{n} \in N$.

In any nilpotent group, the set $\sqrt{S}$ of elements that have powers in a fixed subgroup $S$ is a subgroup. It follows that, for every subgroup $M<N$, the map $M \rightarrow \sqrt{j_{N}(M)}$ satisfies properties (a) to (c). Thus we may identify $M^{*}$ with $\sqrt{j_{N}(M)}<N^{*}$. If $M<N$ has finite index, then $M^{*}=\sqrt{j_{N}(M)}=N^{*}$. And $\left(N^{*}\right)^{\prime}=\left(N^{\prime}\right)^{*}$.

With these facts in hand, for all subgroups of finite index $G_{n}<G$ we have $\left(G_{n}^{\prime}\right)^{*} \supseteq\left(\left(G_{n} \cap N\right)^{\prime}\right)^{*}=\left(\left(G_{n} \cap N\right)^{*}\right)^{\prime}=\left(N^{*}\right)^{\prime}=\left(N^{\prime}\right)^{*}$. Thus $\left(G_{n}^{\prime} N^{\prime}\right)^{*}=\left(G_{n}^{\prime}\right)^{*}$, and from (c) we deduce that $G_{n}^{\prime}\left(N^{\prime} \cap G_{n}\right) / G_{n}^{\prime}$ is torsion. As $G_{n}^{\prime}\left(N^{\prime} \cap G_{n}\right) / G_{n}^{\prime}$ is the kernel of the canonical epimorphism $G_{n} / G_{n}^{\prime} \rightarrow G_{n} N^{\prime} / G_{n}^{\prime} N^{\prime}$, we have

$$
H_{1}\left(G_{n}, \mathbb{Q}\right) \cong\left(G_{n} / G_{n}^{\prime}\right) \otimes \mathbb{Q} \cong\left(G_{n} N^{\prime} / G_{n}^{\prime} N^{\prime}\right) \otimes \mathbb{Q} \cong H_{1}\left(\bar{G}_{n}, \mathbb{Q}\right) \text {. }
$$


Theorem 6.3. Let $N \longmapsto G \rightarrow Q$ be a short exact sequence of groups. If $N$ is nilpotent, $Q$ is abelian and $G$ is of type $\mathrm{FP}_{2}$, then the virtual first betti number $\operatorname{vb}_{1}(G)$ is finite.

Proof. In the light of Proposition 6.2, this follows directly from Theorem 4.3 and the fact [Bieri and Strebel 1980, Theorem 5.5] that $G / N^{\prime}$ is a finitely presented metabelian group.

Corollary 6.4 (Theorem A). If a group $G$ is nilpotent-by-abelian-by-finite and of type $\mathrm{FP}_{2}$, then $\mathrm{vb}_{1}(G)$ is finite.

Proof. Let $G_{0}$ be a subgroup of finite index in $G$ such that $G_{0}$ is nilpotent-by-abelian. Then, $G_{0}$ has type $\mathrm{FP}_{2}$, so $\mathrm{vb}_{1}\left(G_{0}\right)$ is finite, by Theorem 6.3, and hence, so is $G$, by Lemma 4.1 .

Remark 6.5. We did not use the full force of finite presentability in establishing Theorem A: in fact, it is enough to assume that $G$ has a subgroup of finite index $G_{0}$ in which there is a nilpotent subgroup $N \triangleleft G_{0}$ such that $Q=G_{0} / N$ is free abelian and, writing $A=N / N^{\prime}$, the $\mathbb{Q} Q$-module $A \otimes A \otimes \mathbb{Q}$, with diagonal action, should be finitely generated. These requirements follow from the finite presentability of $G_{0} / N^{\prime}$ but are strictly weaker.

Corollary 6.6. Every soluble group of type $\mathrm{FP}_{\infty}$ has finite virtual first betti number. Proof. Soluble groups $S$ of type $\mathrm{FP}_{\infty}$ are constructible and hence nilpotent-byabelian-by-finite [Kropholler 1986].

Corollary 6.7. Every abelian-by-polycyclic group of type $\mathrm{FP}_{3}$ has finite virtual first betti number.

Proof. By the main result of [Groves et al. 2008], abelian-by-polycyclic groups of type $\mathrm{FP}_{3}$ are nilpotent-by-abelian-by-finite.

\section{Acknowledgements}

We thank Fatemeh Mokari and an anonymous referee for helpful comments. We particularly thank the referee for simplifying the proofs of Proposition 6.2 and Claim 2 in Section 3. The work of Prof. Bridson was supported by grants from the EPSRC and by a Wolfson Merit Award from the Royal Society; the work of Prof. Kochloukova was supported by "bolsa de produtividade em Pesquisa", CNPq, Brazil: we thank all of these organizations.

\section{References}

[Abels 1987] H. Abels, Finite presentability of S-arithmetic groups. Compact presentability of solvable groups, Lecture Notes in Math. 1261, Springer, Berlin, 1987. MR 89b:22017 Zbl 0621.20015 [Atiyah and Macdonald 1969] M. F. Atiyah and I. G. Macdonald, Introduction to commutative algebra, Addison-Wesley, Reading, MA, 1969. MR 39 \#4129 Zbl 0175.03601 
[Bieri 1981] R. Bieri, Homological dimension of discrete groups, 2nd ed., Queen Mary College, Department of Pure Mathematics, London, 1981. MR 84h:20047 Zbl 0357.20027

[Bieri and Groves 1985] R. Bieri and J. R. J. Groves, "Tensor powers of modules over finitely generated abelian groups”, J. Algebra 97:1 (1985), 68-78. MR 87c:20017 Zbl 0705.20050

[Bieri and Strebel 1980] R. Bieri and R. Strebel, "Valuations and finitely presented metabelian groups", Proc. London Math. Soc. (3) 41:3 (1980), 439-464. MR 81j:20080 Zbl 0448.20029

[Bieri and Strebel 1981] R. Bieri and R. Strebel, "A geometric invariant for modules over an abelian group”, J. Reine Angew. Math. 322 (1981), 170-189. MR 82f:20017 Zbl 0448.20010

[Bourbaki 1961-1965] N. Bourbaki, Algèbre commutative: chapitres 1-7, Eléments de mathématique 27-28, 30-31, Hermann, Paris, 1961-1965. Translated as Commutative algebra: chapters 1-7, Springer, Berlin, 1998. MR 2001g:13001 Zbl 1103.13001

[Brookes and Groves 1995] C. J. B. Brookes and J. R. J. Groves, "Modules over nilpotent group rings", J. London Math. Soc. (2) 52:3 (1995), 467-481. MR 97f:20009 Zbl 0857.20002

[Brookes and Groves 2000] C. J. B. Brookes and J. R. J. Groves, "Modules over crossed products of a division ring by a free abelian group I", J. Algebra 229:1 (2000), 25-54. MR 2001g:16052 Zbl 0958.16028

[Brookes and Groves 2002] C. J. B. Brookes and J. R. J. Groves, "Modules over crossed products of a division ring by a free abelian group II", J. Algebra 253:2 (2002), 417-445. MR 2003g:16034 Zbl 1011.16018

[Button 2010] J. O. Button, "Largeness of LERF and 1-relator groups", Groups Geom. Dyn. 4:4 (2010), 709-738. MR 2011j:20078 Zbl 1248.20033

[Eisenbud 1995] D. Eisenbud, Commutative algebra: with a view toward algebraic geometry, Graduate Texts in Mathematics 150, Springer, New York, 1995. MR 97a:13001 Zbl 0819.13001

[Groves 1991] J. R. J. Groves, "Some finitely presented nilpotent-by-abelian groups", J. Algebra 144:1 (1991), 127-166. MR 93b:20059 Zbl 0806.20023

[Groves et al. 2008] J. R. J. Groves, D. H. Kochloukova, and C. F. Rodrigues, "Abelian-by-polycyclic groups of homological type $\mathrm{FP}_{3}$ ”, J. Group Theory 11:5 (2008), 609-621. MR 2009j:20074 Zbl 1180.20042

[Kropholler 1986] P. H. Kropholler, "Cohomological dimension of soluble groups", J. Pure Appl. Algebra 43:3 (1986), 281-287. MR 88h:20063 Zbl 0603.20033

[Lackenby 2010] M. Lackenby, "Detecting large groups", J. Algebra 324:10 (2010), 2636-2657. MR 2011h:20045 Zbl 1231.20026

[Malcev 1949] A. I. Malcev, “Нильпотентные группы без кручения", Izvestiya Akad. Nauk. SSSR. Ser. Mat. 13:3 (1949), 201-212. Translated as "Nilpotent torsion-free groups". MR 10,507e Zbl 0034.01702

[McIsaac 1984] A. J. McIsaac, "Some conditions for finite presentation of nilpotent-by-abelian groups", Math. Proc. Cambridge Philos. Soc. 95:3 (1984), 411-415. MR 85k:20104 Zbl 0541.20019

[Quillen 1969] D. Quillen, "Rational homotopy theory”, Ann. of Math. (2) 90 (1969), 205-295. MR 41 \#2678 Zbl 0191.53702

[Robinson and Strebel 1982] D. J. S. Robinson and R. Strebel, "Some finitely presented soluble groups which are not nilpotent by abelian by finite", J. London Math. Soc. (2) 26:3 (1982), 435-440. MR 84m:20041 Zbl 0465.20027

[Wilson 1998] J. S. Wilson, "Finitely presented soluble groups", pp. 296-316 in Geometry and cohomology in group theory (Durham, 1994), edited by P. H. Kropholler et al., London Math. Soc. Lecture Note Ser. 252, Cambridge Univ. Press, 1998. MR 2000m:20052 Zbl 0908.20025 
Received July 25, 2014. Revised September 15, 2014.

MARTIN R. BRIDSON

MATHEMATICAL Institute

UNIVERSITY OF OXFORD

ANDREW WILES BUILDING

WOODSTOCK ROAD

OXFORD

OX2 6GG

UNITED KINGDOM

bridson@maths.ox.ac.uk

Dessislava H. Kochloukova

DEPARTMENT OF MATHEMATICS

UNIVERSITY OF CAMPINAS (UNICAMP)

13083-859 CAMPINAS-SP

BRAZIL

desi@ime.unicamp.br 


\title{
PACIFIC JOURNAL OF MATHEMATICS
}

\author{
msp.org/pjm
}

Founded in 1951 by E. F. Beckenbach (1906-1982) and F. Wolf (1904-1989)

\section{EDITORS}

Don Blasius (Managing Editor)

Department of Mathematics

University of California

Los Angeles, CA 90095-1555

blasius@math.ucla.edu

\author{
Paul Balmer \\ Department of Mathematics \\ University of California \\ Los Angeles, CA 90095-1555 \\ balmer@math.ucla.edu \\ Robert Finn \\ Department of Mathematics \\ Stanford University \\ Stanford, CA 94305-2125 \\ finn@math.stanford.edu \\ Sorin Popa \\ Department of Mathematics \\ University of California \\ Los Angeles, CA 90095-1555 \\ popa@math.ucla.edu
}

\author{
Vyjayanthi Chari \\ Department of Mathematics \\ University of California \\ Riverside, CA 92521-0135 \\ chari@math.ucr.edu \\ Kefeng Liu \\ Department of Mathematics \\ University of California \\ Los Angeles, CA 90095-1555 \\ liu@math.ucla.edu \\ Jie Qing \\ Department of Mathematics \\ University of California \\ Santa Cruz, CA 95064 \\ qing@ cats.ucsc.edu
}

\section{PRODUCTION}

Silvio Levy, Scientific Editor, production@msp.org

\section{SUPPORTING INSTITUTIONS}

ACADEMIA SINICA, TAIPEI

CALIFORNIA INST. OF TECHNOLOGY

INST. DE MATEMÁTICA PURA E APLICADA

KEIO UNIVERSITY

MATH. SCIENCES RESEARCH INSTITUTE

NEW MEXICO STATE UNIV.

OREGON STATE UNIV.

\author{
STANFORD UNIVERSITY \\ UNIV. OF BRITISH COLUMBIA \\ UNIV. OF CALIFORNIA, BERKELEY \\ UNIV. OF CALIFORNIA, DAVIS \\ UNIV. OF CALIFORNIA, LOS ANGELES \\ UNIV. OF CALIFORNIA, RIVERSIDE \\ UNIV. OF CALIFORNIA, SAN DIEGO \\ UNIV. OF CALIF., SANTA BARBARA
}

\author{
Daryl Cooper \\ Department of Mathematics \\ University of California \\ Santa Barbara, CA 93106-3080 \\ cooper@math.ucsb.edu \\ Jiang-Hua Lu \\ Department of Mathematics \\ The University of Hong Kong \\ Pokfulam Rd., Hong Kong \\ jhlu@maths.hku.hk \\ Paul Yang \\ Department of Mathematics \\ Princeton University \\ Princeton NJ 08544-1000 \\ yang@math.princeton.edu
}

These supporting institutions contribute to the cost of publication of this Journal, but they are not owners or publishers and have no responsibility for its contents or policies.

See inside back cover or msp.org/pjm for submission instructions.

The subscription price for 2015 is US \$420/year for the electronic version, and \$570/year for print and electronic.

Subscriptions, requests for back issues and changes of subscribers address should be sent to Pacific Journal of Mathematics, P.O. Box 4163, Berkeley, CA 94704-0163, U.S.A. The Pacific Journal of Mathematics is indexed by Mathematical Reviews, Zentralblatt MATH, PASCAL CNRS Index, Referativnyi Zhurnal, Current Mathematical Publications and Web of Knowledge (Science Citation Index).

The Pacific Journal of Mathematics (ISSN 0030-8730) at the University of California, c/o Department of Mathematics, 798 Evans Hall \#3840, Berkeley, CA 94720-3840, is published twelve times a year. Periodical rate postage paid at Berkeley, CA 94704, and additional mailing offices. POSTMASTER: send address changes to Pacific Journal of Mathematics, P.O. Box 4163, Berkeley, CA 94704-0163.

PJM peer review and production are managed by EditFLOW ${ }^{\circledR}$ from Mathematical Sciences Publishers.

\section{PUBLISHED BY}

\section{mathematical sciences publishers \\ nonprofit scientific publishing}

http://msp.org/

(C) 2015 Mathematical Sciences Publishers 


\section{PACIFIC JOURNAL OF MATHEMATICS}

Volume $274 \quad$ No. $2 \quad$ April 2015

On Demazure and local Weyl modules for affine hyperalgebras

Angelo Bianchi, Tiago Macedo and Adriano Moura

On curves and polygons with the equiangular chord property

305

TARik Aougab, Xidian Sun, Serge TabachniKov and

YUWEN WANG

The well-posedness of nonlinear Schrödinger equations in Triebel-type 325 spaces

SHAOLEI RU and JiECHENG CHEN

Hypersurfaces with constant curvature quotients in warped product manifolds

JIE WU and CHAO XIA

The first terms in the expansion of the Bergman kernel in higher degrees

MARTin PUCHOL and JiAlin ZHU

Determinant rank of $C^{*}$-algebras

Guinua Gong, HuAXIN Lin and Yifeng XUE

Motion by mixed volume preserving curvature functions near spheres

437

DAVID HARTLEY

Homomorphisms on infinite direct products of groups, rings and monoids

GEORGE M. BERGMAN

The virtual first Betti number of soluble groups

Martin R. BRIDSON and Dessislava H. KochloukOVA 